\title{
Errata for the paper entitled "Effects of Eccentric Impeller Position on Radial Passive Stability in a Magnetically Levitated Centrifugal Blood Pump with a Double Volute"
}

Shuya SHIDA, Toru MASUZAWA, Masahiro OSA, Ryota SATO: Adv Biomed Eng. 7, pp. 63-71, 2018.

Upon double-checking the experimental setup, the authors discovered that the impeller diameter and radial clearance of the prototype geometry used in the experiment were incorrect. The correct diameter and radial clearance of prototype geometry were $51 \mathrm{~mm}$ and $1.5 \mathrm{~mm}$, respectively, although a diameter of $52 \mathrm{~mm}$ and radial clearance of $1.0 \mathrm{~mm}$ were described in the original paper and these dimensions were used in some calculations. However, inaccuracy in the description of the experimental setting does not affect the results of computational fluid dynamics (CFD) analysis. Thus, the conclusion of the original paper remains valid because the main discussion is based on the CFD results. Nevertheless, the following corrections should be noted to avoid misunderstanding and to maintain consistency in the original paper. Specifically, in Fig. 5 of the original version, the pressure heads calculated by CFD for the prototype geometry with an incorrect impeller diameter of $52 \mathrm{~mm}$ (radial clearance of $1.0 \mathrm{~mm}$ ) were compared to the pressure heads obtained experimentally using the prototype geometry that actually had an impeller diameter of $51 \mathrm{~mm}$ (radial clearance of $1.5 \mathrm{~mm}$ ). This figure has been amended [see Fig. 5 (corrected version)], in which the CFD-based pressure heads were calculated using the prototype geometry with an impeller diameter of $51 \mathrm{~mm}$ (radial clearance of 1.5

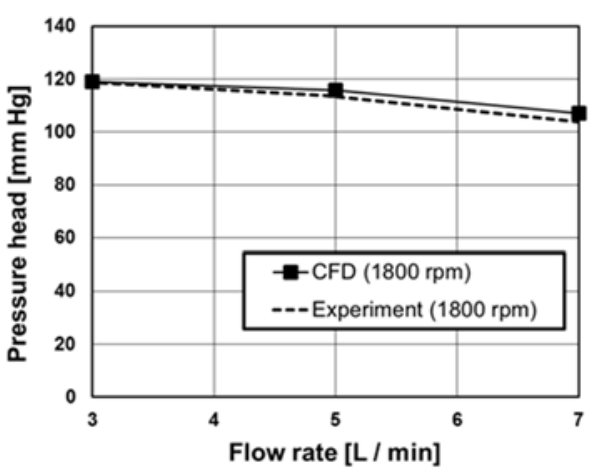

Fig. 5 (corrected version) $\mathrm{mm}$ ). Note that there is no significant difference between the original and corrected versions. Other corrections in the text are tabulated as follows:

\begin{tabular}{|c|c|c|}
\hline Page, section, line & Corrected points in original paper & Description of correction \\
\hline $\begin{array}{l}\text { Page } 64, \text { section } 2.1, \\
\text { paragraph } 1 \text {, lines } 11-12\end{array}$ & "diameter of 52 mm" & "diameter of $51 \mathrm{mm"}$ \\
\hline $\begin{array}{l}\text { Page } 64, \text { section } 2.1, \\
\text { paragraph } 1 \text {, line } 13\end{array}$ & $\begin{array}{l}\text { Additional sentence before the last } \\
\text { sentence }\end{array}$ & "The radial clearance was $1.5 \mathrm{~mm} . "$ \\
\hline $\begin{array}{l}\text { Page } 65, \text { section } 2.2, \\
\text { paragraph } 1 \text {, line } 4\end{array}$ & $\begin{array}{l}\text { Additional sentences after the } \\
\text { sentence, "The geometric features } \\
\text { are listed in Table 1." }\end{array}$ & $\begin{array}{l}\text { "The outer diameter of the impeller was } 52 \\
\text { mm for all geometries in the CFD analysis. } \\
\text { A radial clearance of } 1 \mathrm{~mm} \text { was used in the } \\
\text { CFD analysis of the prototype geometry to } \\
\text { adjust the analysis setting to that of the } \\
\text { H6DL series." }\end{array}$ \\
\hline Page 65, Table 1 heading & "for this study" & "for this CFD analysis" \\
\hline $\begin{array}{l}\text { Page } 66 \text {, section } 3.1 \text {, } \\
\text { paragraph } 1 \text {, lines } 2-3\end{array}$ & "Displacement of $1 \mathrm{~mm} "$ & "Displacement of $1.5 \mathrm{~mm} "$ \\
\hline $\begin{array}{l}\text { Page } 66 \text {, section } 3.2, \\
\text { paragraph } 1 \text {, line } 2\end{array}$ & "the prototype geometry" & $\begin{array}{l}\text { "the prototype geometry with an impeller } \\
\text { diameter of } 51 \mathrm{~mm} \text { and a radial clearance of } \\
1.5 \mathrm{~mm} \text { " }\end{array}$ \\
\hline $\begin{array}{l}\text { Page } 66, \text { section } 3.2, \\
\text { paragraph } 1 \text {, line } 4\end{array}$ & "1.8\% greater" & “3.7\% smaller" \\
\hline
\end{tabular}

